\title{
Site-specific modification of ED-B-targeting antibody using intein-fusion technology
}

Sina Möhlmann ${ }^{1}$, Peter Bringmann ${ }^{2}$, Simone Greven ${ }^{1}$ and Axel Harrenga ${ }^{3^{*}}$

\begin{abstract}
Background: A promising new approach in cancer therapy is the use of tumor specific antibodies coupled to cytotoxic agents. Currently these immunoconjugates are prepared by rather unspecific coupling chemistries, resulting in heterogeneous products. As the drug load is a key parameter for the antitumor activity, site-specific strategies are desired. Expressed protein ligation (EPL) and protein trans-splicing (PTS) are methods for the specific C-terminal modification of a target protein. Both include the expression as an intein fusion protein, followed by the exchange of the intein for a functionalized moiety.

Results: A full-length IgG specific for fibronectin ED-B was expressed as fusion protein with an intein (Mxe GyrA or Npu DnaE) attached to each heavy chain. In vitro protocols were established to site-specifically modify the antibodies in high yields by EPL or PTS, respectively. Although reducing conditions had to be employed during the process, the integrity or affinity of the antibody was not affected. The protocols were used to prepare

immunoconjugates containing two biotin molecules per antibody, attached to the C-termini of the heavy chains.

Conclusion: Full-length antibodies can be efficiently and site-specifically modified at the C-termini of their heavy chains by intein-fusion technologies. The described protocols can be used to prepare immunoconjugates of high homogeneity and with a defined drug load of two. The attachment to the C-termini is expected to retain the affinity and effector functions of the antibodies.
\end{abstract}

\section{Background}

Monoclonal antibodies have been approved as therapeutic agents for indications including viral infections, immunological disorders, transplant rejection and cancer [1]. They often act by blocking the function of their target molecule. More demanding is the therapy of cancer by antibodies requiring the specific recognition and subsequent elimination of tumor cells.

Several mechanisms have been described how therapeutic antibodies elicit cell death, including the triggering of apoptosis and the recruitment of the immune system. While therapeutic antibodies have been approved working by these mechanisms (e.g. Rituximab [2], Trastuzumab [3], Alemtuzumab [4]) their cytotoxic potential is generally not sufficient to completely eliminate the malignant cells. Higher efficacies have been observed if the antibody is coupled to toxic agents like radioisotopes (radioimmunoconjugates) or chemical drugs (antibody-

\footnotetext{
* Correspondence: axel.harrenga@bayer.com

${ }^{3}$ Bayer Healthcare, Nattermannallee 1, 50829 Cologne, Germany

Full list of author information is available at the end of the article
}

drug-conjugates, ADC) [5]. Several of these conjugates have been approved for cancer (Ibritomomab, Tositumomab) or are in clinical development (e.g. TrastuzumabDM1).

Coupling of toxic agents to therapeutic antibodies also paves the way for new tumor associated antigens as these are not required to be present on the surface of the malignant cells. An example is the extra domain B (ED-B) of fibronectin, a protein of the extracellular matrix. ED-Bcontaining fibronectin is a splice variant associated with angiogenesis and tissue remodeling [6]. High levels of ED$B$ expression have been detected in most solid tumors and in vivo studies with ED-B specific monoclonal antibody formats show the selective accumulation in tumors and metastases. Accordingly, ED-B is a promising target for antibody-based cancer treatment $[7,8]$ and the results of first clinical trials with ED-B specific antibody fragment conjugates are encouraging $[9,10]$.

Current methods for the preparation of immunoconjugates rely on the chemical coupling to lysine, cysteine or tyrosine side chains [11]. These methods are rather
C Biomed Central

(ㄷ) 2011 Möhlmann et al; licensee BioMed Central Ltd. This is an Open Access article distributed under the terms of the Creative Commons Attribution License (http://creativecommons.org/licenses/by/2.0), which permits unrestricted use, distribution, and reproduction in any medium, provided the original work is properly cited. 
unspecific and result in heterogeneous products. As the drug load - number of toxophore per antibody - is a key parameter for the antitumor activity of immunoconjugates [12-14] more site-specific coupling reactions are desired. Approaches employing the carbohydrate moieties $[15,16]$, the $\mathrm{N}$ - and the C-terminus $[17,18]$ of fulllength IgG antibodies have been described. However, the carbohydrates are important for the effector functions of the Fc domain [19] and the N-terminus of antibodies is close to their antigen binding site which may result in decreased affinity after modification. This leads to the Cterminus as a preferred site for specific drug attachment.

Several enzymatic approaches have been described for the modification of protein C termini [20]. They have in common that the target protein is expressed in fusion with a $\mathrm{C}$-terminal tag containing the modification site. A common drawback of these methods is an incomplete conversion. Without the possibility for separation, this would result in heterogeneous preparations of low averaged drug loads. Interestingly, the intein tag is cleaved off from the target protein during modification, facilitating preparative separation of modified from non-modified protein. Inteins encompass catalytic domains which lead to the formation of a thioester bond at their junction to the target protein. This thioester bond can be employed to exchange the intein for a C-terminal probe. The probe is eventually connected via a native peptide bond [21].

Methods for intein-mediated C-terminal protein modification encompass expressed protein ligation (EPL) and protein trans-splicing (PTS). In EPL, the target protein is fused to a modified full-length intein. The intein is cleaved off by the addition of a thiol reagent, leaving a thioester bond (first step), and the target protein is ligated to a probe functionalized with an $\mathrm{N}$-terminal cysteine residue (second step) [22,23]. In PTS, inteins are used which are split into two parts with high affinity to each other. The large N-terminal part is fused to the target protein. The probe is functionalized with the small C-terminal part of the intein. Their combination results in a functional intein, which splices itself out and concomitantly fuses the target protein to the probe [24-26].

Intein-fusion technologies have already been used for several applications, including the derivatization of small single-chain and single-domain antibody formats with fluorophores and micelles, respectively [27-29]. Here we explored the use of two distinct inteins for $\mathrm{C}$-terminal modification of a full-length antibody. The first is the GyrA intein from Mycobacterium xenopi (Mxe) which is a 'minimal' intein of 198 residues [30]. It can be used for protein modification by EPL [31]. The second is the split DnaE intein from Nostoc punctiforme (Npu) [32,33]. It consists of an $\mathrm{N}$-terminal part of 102 residues and a C-terminal of 36 residues. Npu DnaE was employed for protein modification by PTS. Each intein was fused to the heavy chain of anti ED-B IgG1, resulting in two inteins per antibody (Figure 1). The fusion proteins were expressed by secretion from transfected human embryonic kidney (HEK) cells. After purification, in vitro cleavage by $M x e$ GyrA or trans-splicing by Npu DnaE yielded $60 \%$ or $80 \%$, respectively, of completely processed antibody. The protocols described resulted in functional antibodies and were used to generate immunoconjugates containing two biotin moieties per protein molecule, attached site-specifically to the C-termini of the heavy chains.

\section{Results and Discussion}

\section{Production of L19 IgG antibodies with C-terminal intein} domains

Antibodies require an oxidizing environment for their expression as they contain several disulfide bonds important for proper folding. In addition, glycosylation has been shown to be important for the effector functions of antibodies [19]. They are therefore usually produced by mammalian expression systems. We chose transiently transfected HEK293-6E cells for the expression of L19 IgG antibody which is specific for the ED-B domain of fibronectin. Endogenous signal sequences ensure the secretion into the extracellular medium and therefore the proper folding of the immunoglobulin domains. Connected via an 11 residue linker, the heavy chain was fused to an intein domain. We tested two distinct inteins, GyrA from Mycobacterium xenopi [31] and the split intein DnaE from Nostoc punctiforme [32]. In case of DnaE, L19 IgG was fused to the $\mathrm{N}$-terminal domain $\mathrm{DnaE}_{\mathrm{N}}$ (residues 1-102). Both inteins were extended C-terminally by a decahistidine tag for purification purposes.

Antibody-intein fusion proteins were obtained in good yields (90-110 mg/L). They were purified by His tag affinity chromatography and subsequent gel filtration. A common problem with GyrA fusion proteins is the occurence of intein cleavage during expression. This in vivo cleavage decreases the product yield and is detectable by the copurification of the separated intein domain after His Tag affinity chromatography (Figure 2). However, judged by the subsequent gel filtration elution profile (not shown), only $10-15 \%$ in vivo cleavage was observed for GyrA.

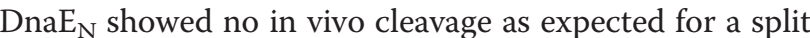
intein.

\section{Preparation of immunoconjugates by Mxe GyrA intein mediated EPL}

In vitro cleavage of L19 IgG-GyrA fusion protein was induced by the addition of $50 \mathrm{mM}$ Mesna which has been reported to form a long-living thioester at the $\mathrm{C}$ terminus. This thioester can be employed to link to the IgG a probe that is functionalized with an $\mathrm{N}$-terminal cysteine residue. It is added concomitantly with Mesna but in lower concentration $(5 \mathrm{mM})$. The cysteine replaces 


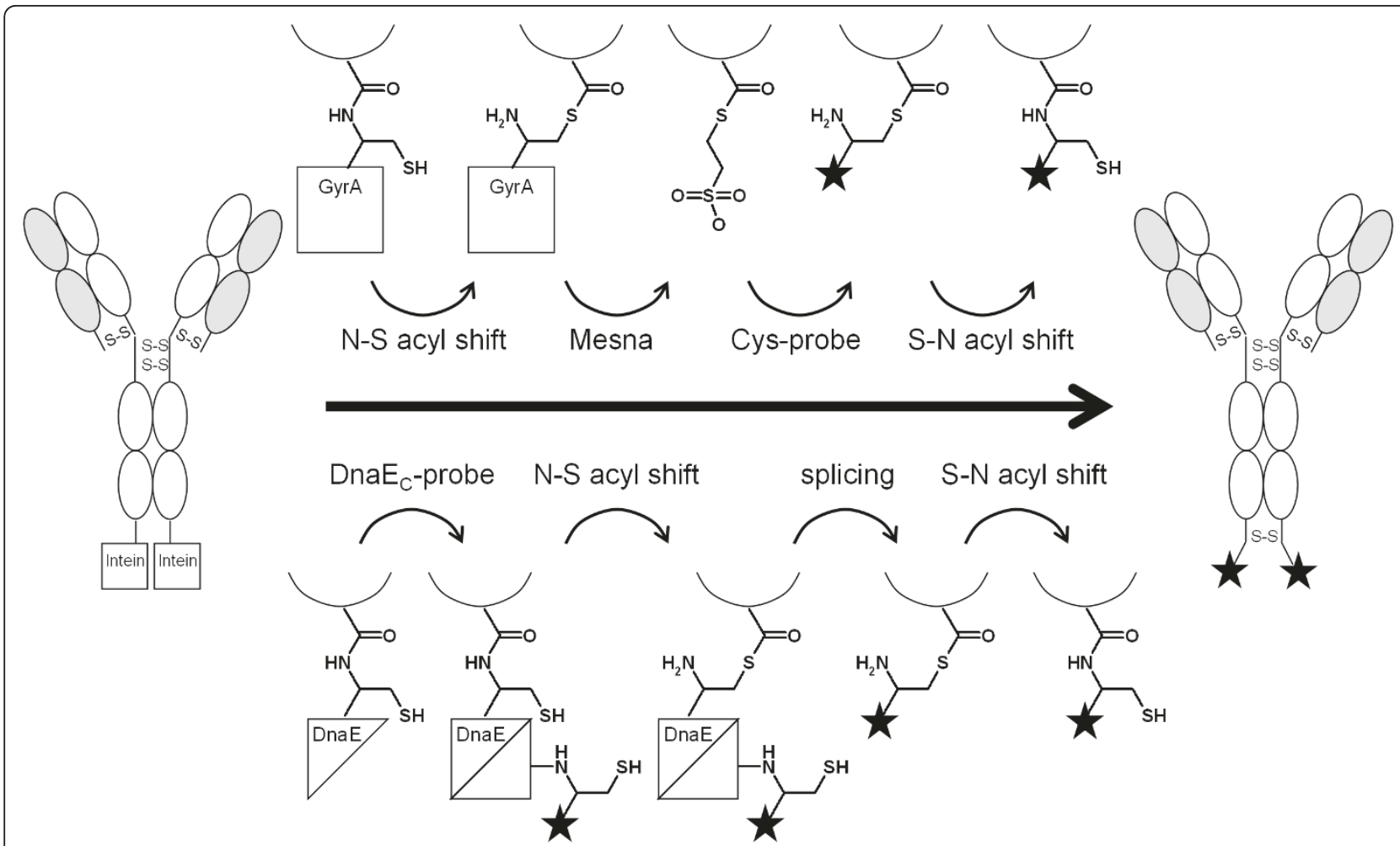

Figure 1 Illustration of chemical steps during in vitro processing of IgG-intein fusion protein. Above the arrow, the chemistry of GyrA intein is shown. After intein-catalyzed N-S acyl shift, GyrA is substituted by Mesna. Mesna-thioester is cleaved by the probe functionalized with an $\mathrm{N}$-terminal cysteine residue, and finally rearranges to a stable peptide bond. Below the arrow, the chemistry of DnaE intein is shown. The intein has to be activated by association of its $\mathrm{N}$ - and C-terminal part. The probe is attached to the C-terminal part and transferred after the intein-catalyzed N-S acyl shift.

the Mesna-thioester, resulting in a stable peptide bond between the IgG and the probe (Figure 1).

To study the cleavage efficiency of the L19 IgG-GyrA fusion protein and to test the antibody integrity after EPL, cysteine was used as 'probe'. After 22h, Mesna and cysteine were removed by intensive dialysis to regenerate and stabilize the disulfide bonds of the antibody. L19 IgG cleaved on both heavy chains could be separated from non- or partly-cleaved antibody by His Tag affinity chromatography (Figure 3). The yield of completely cleaved antibody was about $60 \%$. To test if its functionality had been damaged by the reducing conditions used during EPL it was intensively analyzed. SDS-PAGE under non-reducing conditions resulted in a single band at $150 \mathrm{kDa}$ (calculated: $146.3 \mathrm{kDa}$ ), showing that interchain disulfide bonds were intact (Figure 4A). Analytical gel filtration confirmed the integrity of the antibody, eluting in a single peak very similar to unmodified L19 IgG (control antibody) (Figure 4B). Finally, interaction studies by Surface Plasmon Resonance (SPR) were employed to evaluate antibody functionality after EPL. The antigen binding part was analyzed by investigation of the interaction with the ED-B domain. Binding kinetics and the dissociation constant ( $90 \mathrm{nM})$ were the same as for the L19 IgG control antibody (Figure 4C). To test the functionality of the Fc part, the interaction with the high affinity Fc $\gamma$ RI/CD64 and with the low affinity Fcy RIIIA/CD16a was analyzed by SPR. Binding kinetics and the dissociation constants for both Fc $\gamma$ receptors (Fcy RI/CD64: $0.2 \mathrm{nM}$; Fc $\gamma$ RIIIA/CD16a: $\sim 60 \mathrm{nM}$ ) did not change significantly demonstrating preservation of full functionality (see Additional file 1). In summary, these results show that antigen binding as well as effector functions of L19 IgG have not been affected by the EPL process.

A side reaction of EPL is the hydrolysis of the thioester instead of its derivatization with cysteine. To estimate the yield of L19 IgG-Cys, a pepsin digest assay was employed. Pepsin can be used to prepare Fab dimers from an IgG1 as it cleaves $\mathrm{C}$-terminal to the interchain disulfide bonds connecting the heavy chains [34]. The Fc part of the antibody is cleaved into several fragments. Mass spectrometry analysis of L19 IgG control antibody after pepsin digest ( $\mathrm{pH} 4.5$ ) revealed that the most $\mathrm{C}$-terminal cleavage site is the linker between $\mathrm{CH} 2$ and $\mathrm{CH} 3$ domain, leaving the $\mathrm{CH} 3$ domain intact (Figure 5A). This could 


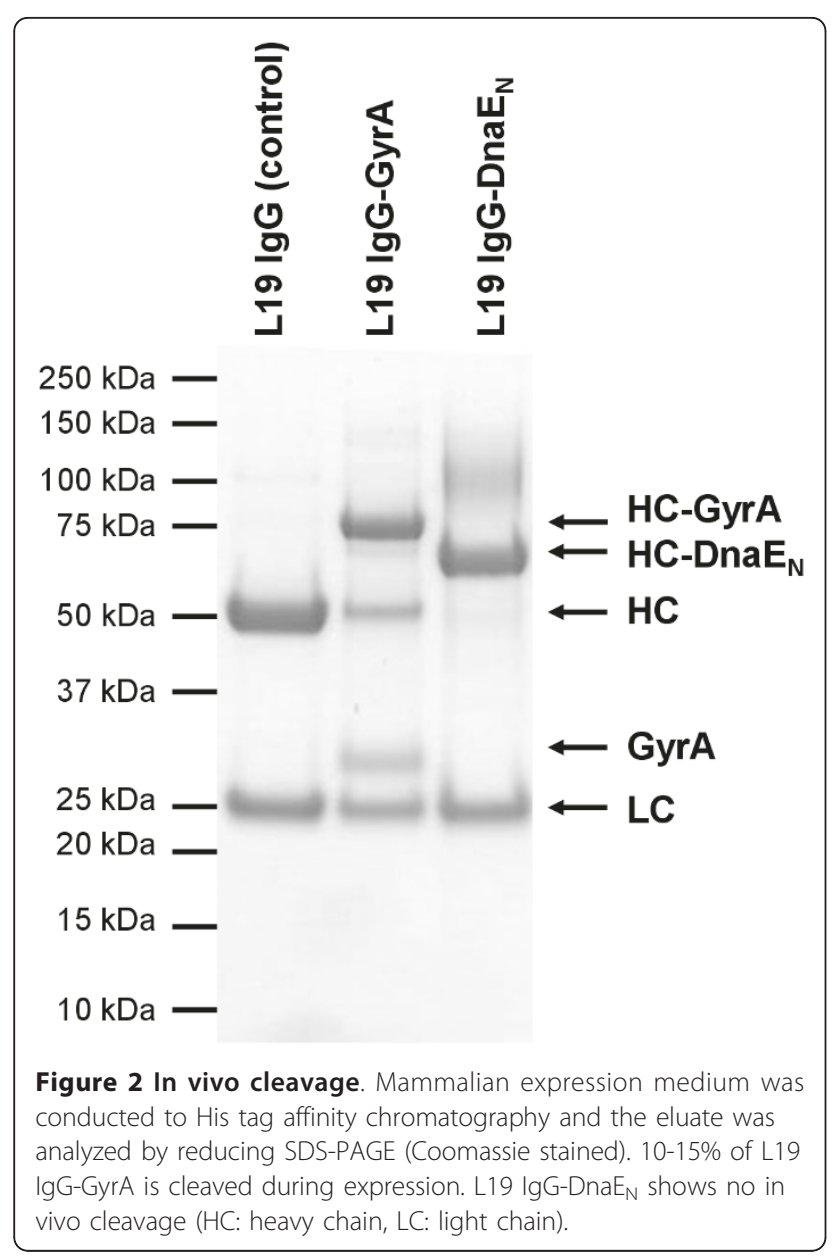

be used for the observation of cysteine derivatization: If cysteines are attached $\mathrm{C}$-terminal to the heavy chains they are expected to form an interchain disulfide bond, resulting in a $28 \mathrm{kDa}$ fragment under non-reducing conditions. However, if one or both cysteines are missing, 14 $\mathrm{kDa}$ fragments are observed. Non-reducing SDS-PAGE after pepsin digest of L19 IgG-Cys revealed the occurrence of a $28 \mathrm{kDa}$ band which was replaced by a $14 \mathrm{kDa}$ band after reduction (Figure $5 \mathrm{~B}$ ). Both bands were excised from the gel and analyzed after tryptic digest with HPLC ESI-Q-TOF. Clearly, only peptides from the $\mathrm{CH} 3$ domain were found (Figure 5C). Also the C-terminal peptide was observed, carrying the cysteine. Interestingly, the $14 \mathrm{kDa}$ band is hardly detectable on the nonreducing SDS-PAGE gel. Mass spectrometry analysis of a non-reduced sample after pepsin digest confirmed this observation: We detected a $28 \mathrm{kDa}$ fragment but no 14 $\mathrm{kDa}$ fragment (Figure 5C). We conclude that the fraction of hydrolyzed protein is minor and that cysteine derivatized L19 IgG was obtained in high yield. This would result in a homogeneous drug load of two. In addition, a $\mathrm{C}$-terminal disulfide bond is readily formed. This may

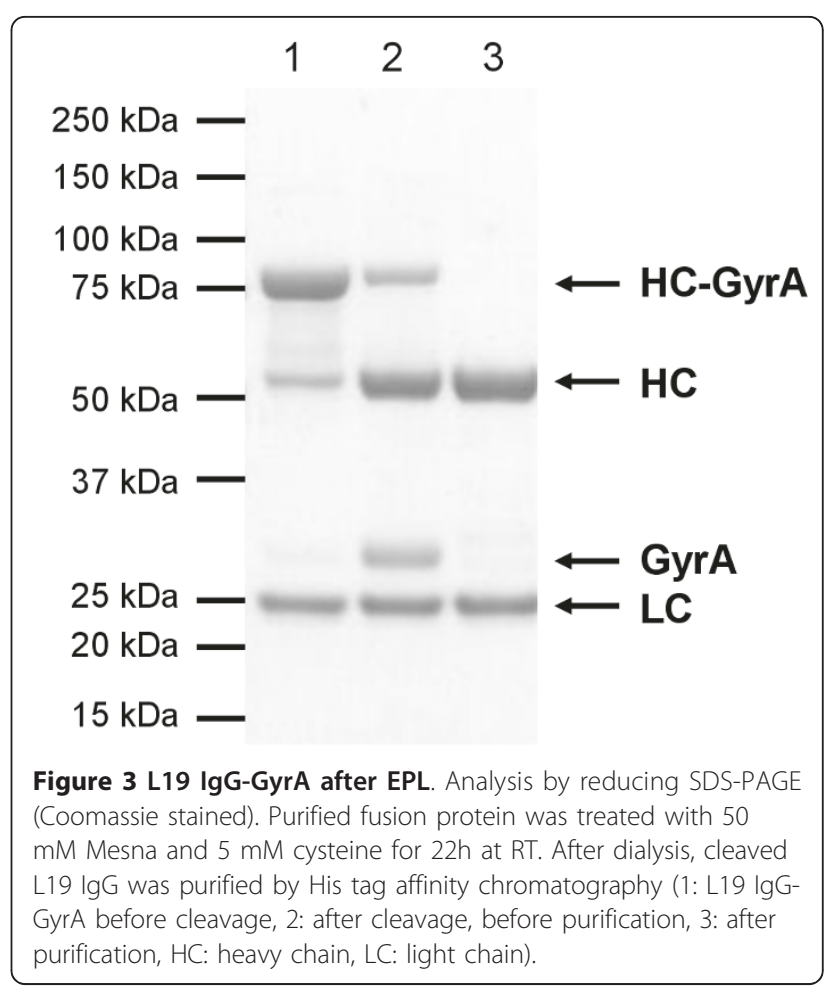

stabilize the immunoconjugate and hinders undesired side reactions of thiol groups in the oxidizing environment antibodies are held.

The aim of our work was to use GyrA intein for the attachment of probes functionalized with an $\mathrm{N}$-terminal cysteine residue. To show the usability of the protocol for this purpose, a CysLys-Biotin peptide was added instead of cysteine. After SDS-PAGE and Western blotting, the specific attachment of biotin to the heavy chain was verified by Streptavidin-based staining (Figure 6). In summary, the described EPL protocol is applicable for the site specific modification of full-length IgG by probes attached to an $\mathrm{N}$-terminal cysteine residue.

\section{Preparation of immunoconjugates by $\mathrm{Npu}$ DnaE intein catalyzed PTS}

L19 IgG-DnaE $\mathrm{N}_{\mathrm{N}}$ contains an incomplete intein which was activated in vitro by the addition of a peptide comprising the missing $\mathrm{C}$-terminal 36 residues, followed by the naturally occurring post-intein sequence CFN. This $\mathrm{DnaE}_{C}$ peptide can be employed to transfer a C-terminally attached probe to L19 IgG by PTS (Figure 1). Importantly, trans-splicing was only observed under reducing conditions i.e. after the addition of $5 \mathrm{mM}$ DTT. We propose that this is due to the requirement of a free cysteine in the reactive center of DnaE intein.

To establish the PTS protocol, DnaE $_{C}$ peptide without a probe was used. After $24 \mathrm{~h}$ trans-splicing reaction, DTT was removed by dialysis and completely spliced 


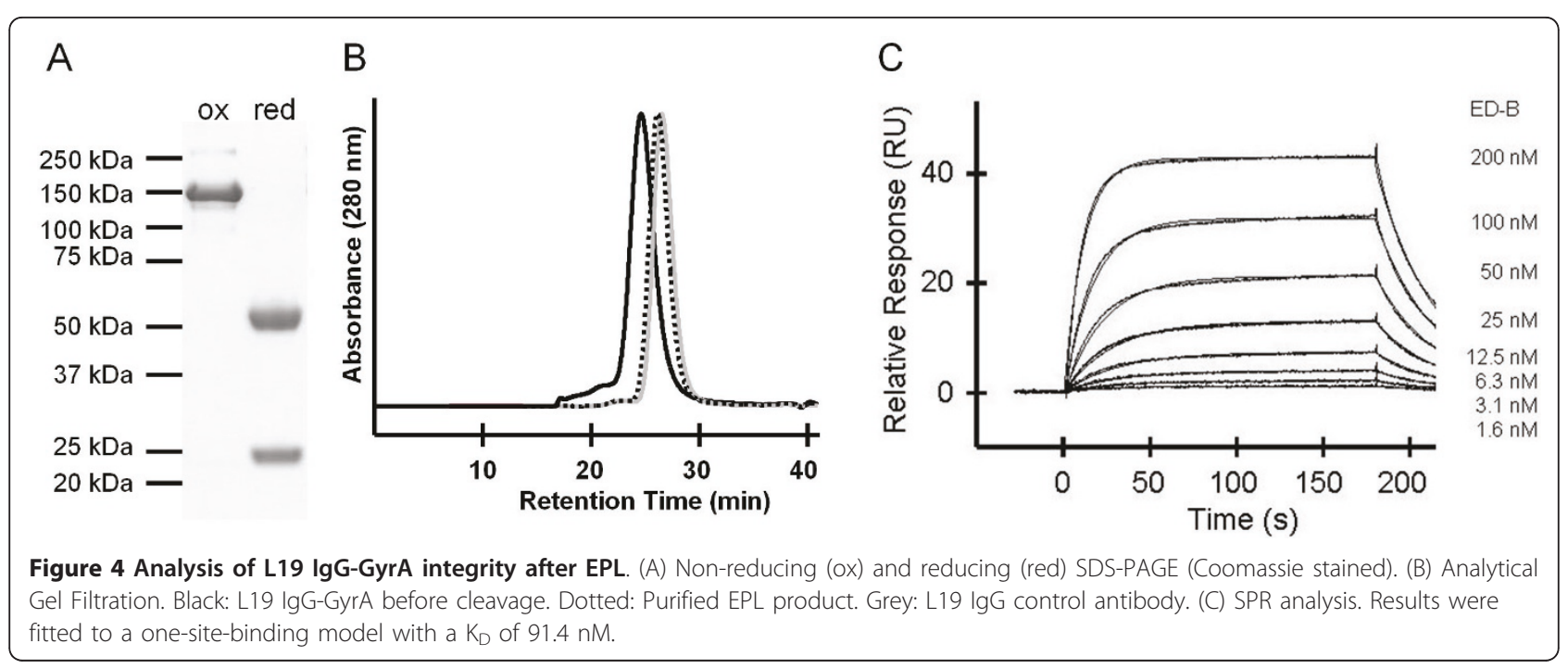

L19 IgG was purified by His tag affinity chromatography (Figure 7). The yield of L19 IgG processed on both heavy chains was about $80 \%$. Analysis were undertaken to test if the antibody had been damaged by the reducing conditions used during PTS. SDS-PAGE under non-reducing conditions showed that interchain disulfide bonds were intact as a main band at $150 \mathrm{kDa}$ (calculated: $146.9 \mathrm{kDa}$ ) was observed (Figure 8A). However, a weak band corresponding to a protein species with an apparent molecular mass of $300 \mathrm{kDa}$ indicated the presence of a small amount of antibody dimers. These results were confirmed by analytical gel filtration (Figure 8B). L19 IgG-DnaE $\mathrm{N}_{\mathrm{N}}$ trans-splicing product eluted in a main peak very similar to unmodified L19 IgG (control antibody) but is accompanied by a small preceding peak typical for dimerization. We conclude that the integrity of L19 IgG is retained after PTS. The small percentage of dimerization could be due to some degree of

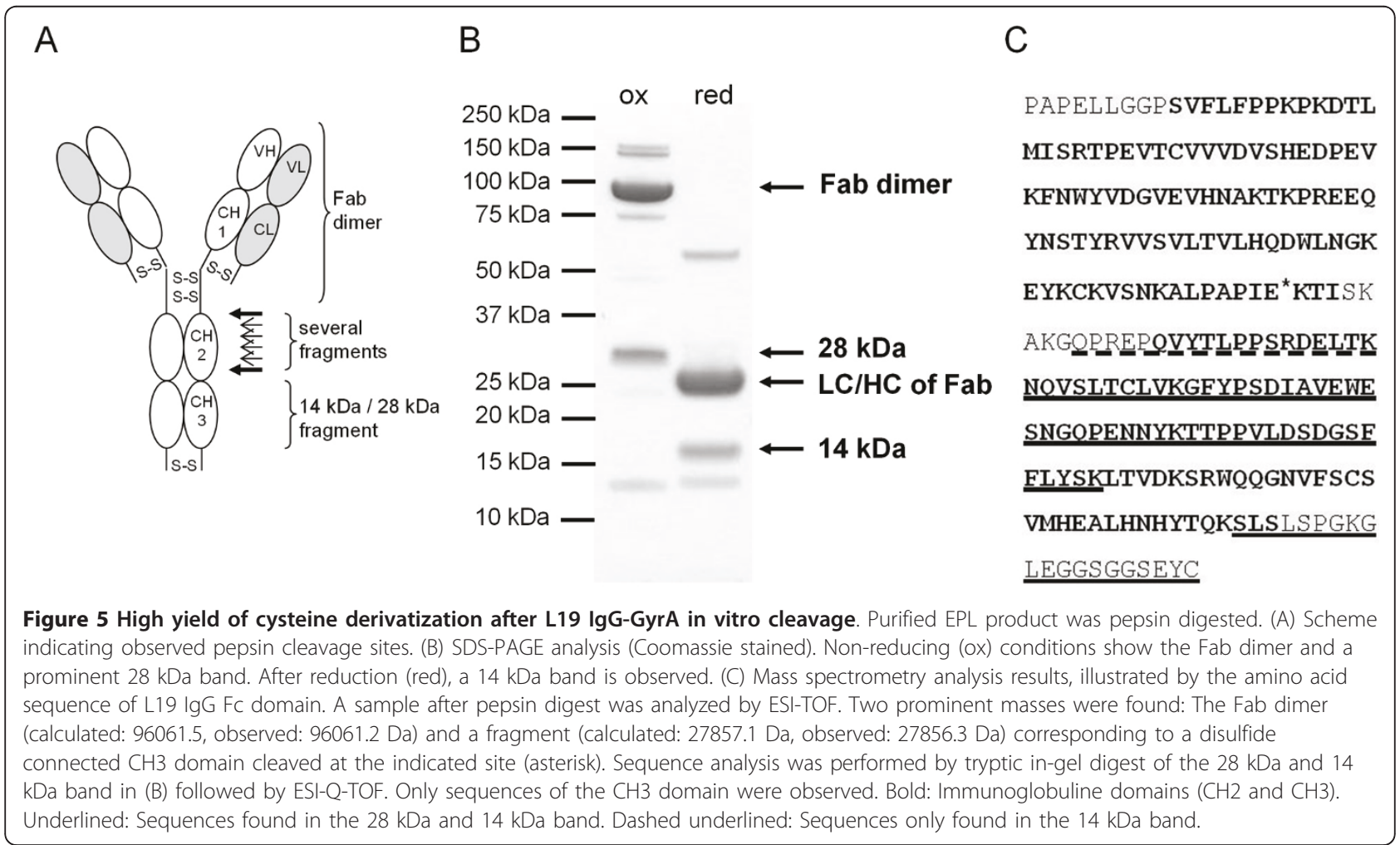




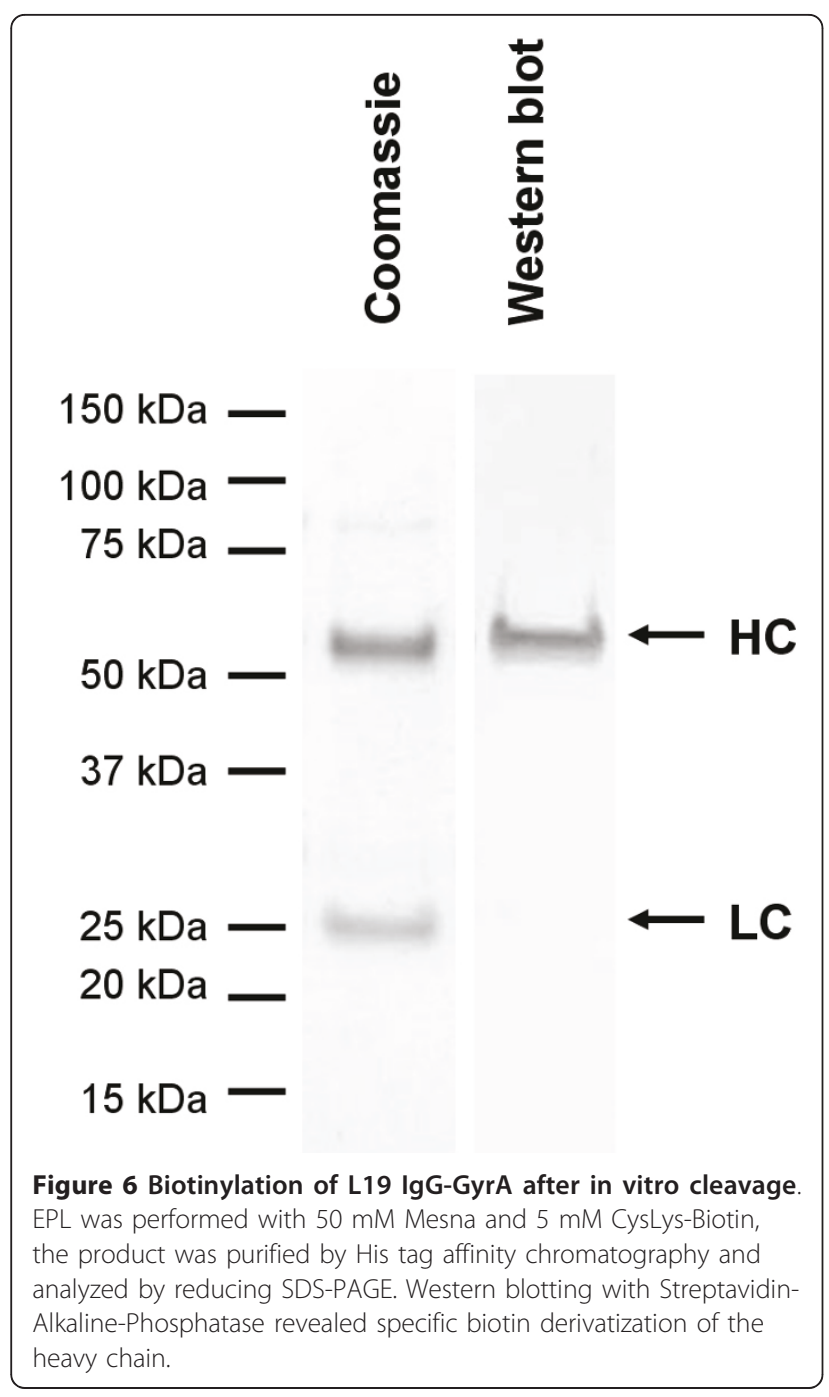

hydrolysis during intein catalyzed trans-splicing (see below). This would result in antibodies with single cysteine residues at the $\mathrm{C}$-terminus which are prone to pairing by disulfide bond formation. To test the functionality of L19 IgG after PTS, its interaction with the ED-B domain was analyzed by SPR. Binding kinetics and the dissociation constant $(\sim 90 \mathrm{nM})$ were the same as for the L19 IgG control antibody (Figure 8C). In addition, the interaction with the high affinity Fc $\gamma$ RI/ CD64 and with the low affinity Fc $\gamma$ RIIIA/CD16a was investigated by SPR. Binding kinetics and the dissociation constants for both Fc $\gamma$ receptors (Fc $\gamma$ RI/CD64: $\sim 0.2 \mathrm{nM}$; Fc $\gamma$ RIIIA/CD16a: 60nM) were not compromised by PTS, demonstrating the full functionality of the Fc domain (see Additional file 1). In summary, our DnaE PTS protocol does neither affect the integrity nor the functionality of L19 IgG.

In the next step we used the DnaE intein to attach biotin probes to the antibody. A functionalized $\mathrm{DnaE}_{\mathrm{C}}$

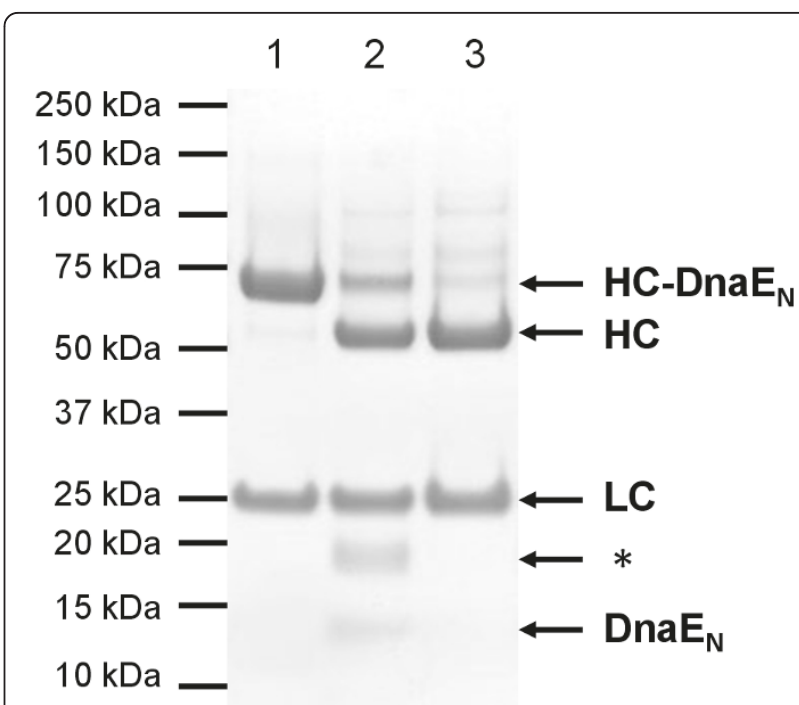

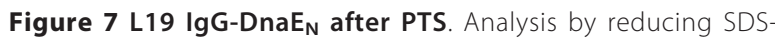
PAGE (Coomassie stained). Purified fusion protein was treated with $5 \mathrm{mM}$ DTT and $50 \mu \mathrm{M} \mathrm{DnaE}$ Ceptide for $22 \mathrm{~h}$ at RT. After dialysis, trans-spliced L19 IgG was purified by His tag affinity chromatography (1: L19 lgG-DnaE before PTS, 2: after PTS, before purification, 3: after purification, HC: heavy chain, LC: light chain, * product not further investigated).

peptide (DnaE ${ }_{C}$-CFNK-Biotin) was applied according to our protocol and the L19 IgG product was analyzed by SDS-PAGE and Western blotting (Figure 9). Biotin was specifically attached to the heavy chain.

The requirement to synthesize a 39 residue peptide $\left(\mathrm{DnaE}_{\mathrm{C}}-\mathrm{CFN}\right)$ makes the described protocols for attachment of small probes more complicated with DnaE than with GyrA. However, as DnaE $\mathrm{C}_{\mathrm{C}}$ begins on a methionine residue, it can easily be expressed in vivo at the $\mathrm{N}$-terminus of a fusion protein. Proteins like exotoxins, RNases or cytokines have been described to be effective against tumor cells if fused to a targeting antibody [35]. In contrast to IgGs, these effector proteins can usually be produced by bacterial expression systems. We suggest that DnaE catalyzed PTS could be used to combine a given antibody with a diversity of effector proteins. To test our protocol for this purpose, $\mathrm{DnaE}_{\mathrm{C}}$ fused to glutathione-Stransferase (GST) was purified from recombinant $E$. coli and incubated with L19 IgG-DnaE ${ }_{\mathrm{N}}$. Analysis by reducing SDS-PAGE showed that a main product of $80 \mathrm{kDa}$ had been formed (Figure 10A). This corresponds to the heavy chain fused to GST (calculated: $76.8 \mathrm{kDa}$ ). Western blotting confirmed the presence of GST in the band (Figure 10B). In summary, our DnaE PTS protocol is suitable to obtain L19 IgG antibody functionalized with probes or effector proteins in good yield.

L19 IgG heavy chain conjugated to GST $(80 \mathrm{kDa})$ was the main product after PTS with $\mathrm{DnaE}_{\mathrm{C}}-\mathrm{GST}$ as probe. 

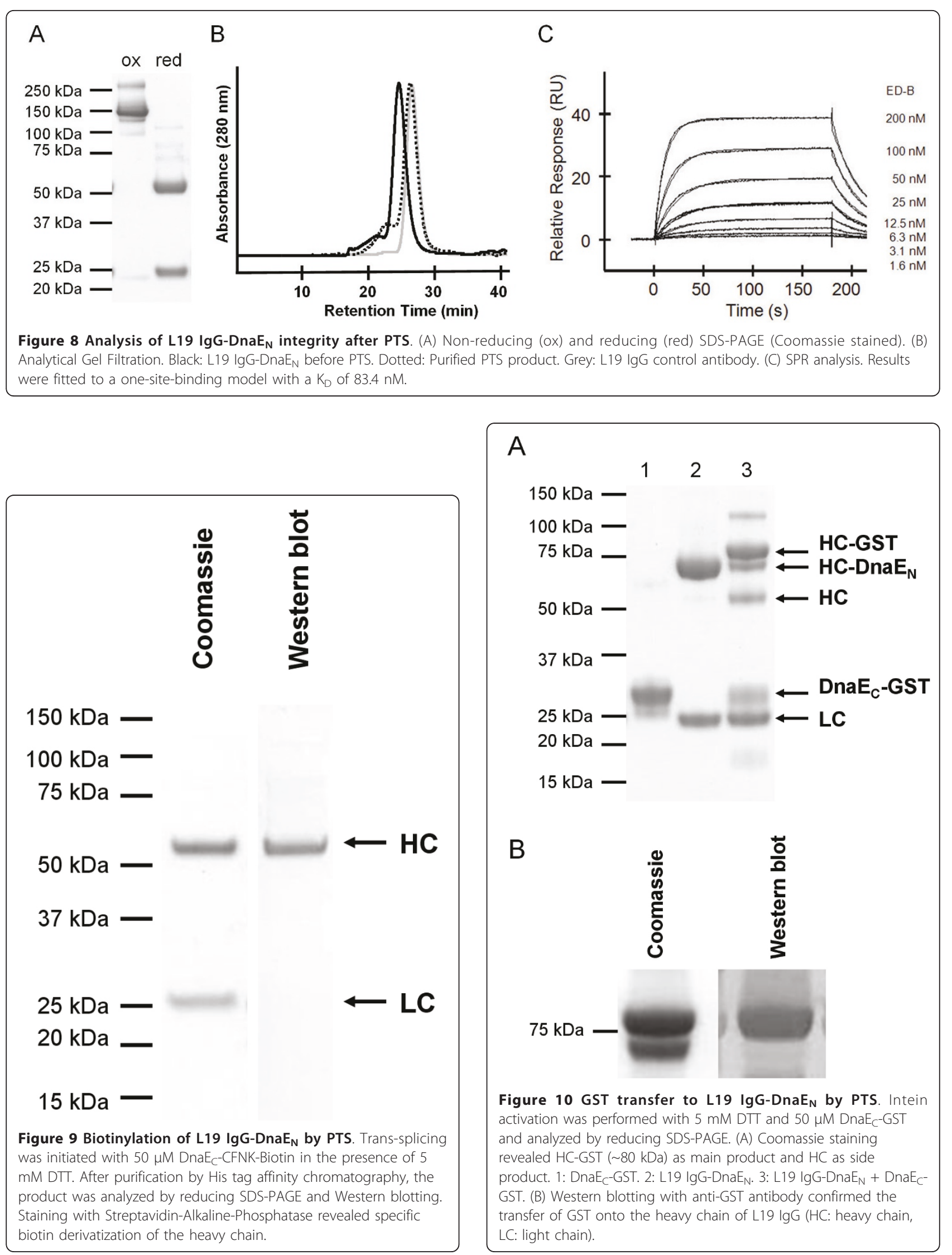
However, also a side product of $50 \mathrm{kDa}$ was observed. It corresponded to the heavy chain of L19 IgG produced by hydrolysis of the thioester intermediate formed during intein catalysis and allowed evaluation of its magnitude compared to probe conjugation. We estimate from the gel that about $25 \%$ of processed heavy chain was hydrolyzed while about $75 \%$ encompassed the desired conjugation product. Due to this hydrolysis, the drug load of immunoconjugates prepared by our DnaE PTS protocol reached only about 1.5 . It is likely that hydrolysis was promoted by attack of the thiol reagent DTT on the intein-derived thioester [33]. DTT had to be added to ensure reducing conditions for optimal intein activity. Alternative reducing agents like tris(2-carboxyethyl) phosphine (TCEP) could decrease hydrolysis and further optimize the PTS protocol.

\section{Conclusions}

We have shown here for the first time that full-length IgGs can be specifically functionalized at the C-terminus of their heavy chains by EPL and PTS. Antibodies processed on both chains were obtained in good yields and purified from incompletely processed proteins. The attachment of two probes per antibody and in vicinity to each other yielded an additional disulfide bond, circumventing problems with free thiol groups introduced by inteinfusion technologies. These methods could be employed to generate immunoconjugates of high homogeneity and with a drug load of two. The modification at the C-termini of the heavy chains does not interfere with antigen binding and, judged by binding to Fc $\gamma$ RI and Fc $\gamma$ RIIIA, it does not compromise the effector functions of the Fc domain. Provided with an effective toxophore, this would result in therapeutic antibodies fully equipped for the elimination of malignant cells.

\section{Methods}

\section{General}

Chemicals were purchased from Sigma Aldrich if not stated otherwise. Chromatography material (Glutathione Sepharose 4 Fast Flow, HiTrap Chelating HP, Superdex 200) was obtained from GE Healthcare (Munich).

\section{Vectors}

L19 IgG was derived by transferring the variable domains of L19 scFv [36] into a human IgG1/к format.

Mxe GyrA (from vector pTXB1, New England Biolabs) or $N p u$ DnaE $_{\mathrm{N}}$ (from vector pSKDuet, provided by Hideo Iwai, University of Helsinki) were cloned behind the heavy chain of L19 IgG, separated by a linker coding for GLEGGSGGSEY. GyrA and DnaE ${ }_{N}$ were extended C-terminally to include a His tag $\left(\mathrm{GSA}-\mathrm{H}_{10}\right.$ and $\mathrm{AS}-\mathrm{H}_{10}$, respectively). Constructs encoding L19 IgG (control, without linker) and L19 IgG fused to GyrA or DnaE $E_{N}$ were transferred into pTT5 vector (National Research Council Canada). In the resulting expression vectors, both, heavy and light chain, were under the control of an hCMV promoter.

DNA encoding DnaE $_{C}$ from Nostoc punctiforme (MIKIATRKYLGKQNVYDIGVERDHNFALKNGFIASN [32]), followed by a linker (CFNPAGSSGVIM) and Glutathione-S-Transferase (GST, Schistosoma japonicum) was purchased from Geneart (Regensburg, Germany), amplified by PCR and ligated into vector pET29a (Novagen) by NdeI/NotI.

\section{Expression and Purification of L19 IgG constructs}

0.5 L of HEK293-6E cells were grown in F17 medium with 4 mM GlutaMAX-I and 0.1\% Pluronic F-68 (Invitrogen) to a density of $1.5-2.0 \times 10^{6}$ cells $/ \mathrm{mL}$ and transfected with $500 \mu \mathrm{g}$ plasmid DNA (L19 IgG constructs) as described [37], with minor modifications. After five days cells were harvested by centrifugation $\left(8000 \times \mathrm{g}, 5^{\prime}\right)$. The supernatant was concentrated to $50 \mathrm{~mL}$ by crossflow filtration (Sartorius) and dialyzed against column buffer (500 mM NaCl, 50 mM Hepes-NaOH, pH 7.5). For purification, Ni-loaded IMAC (elution with imidazole gradient) and subsequent gel filtration was applied.

\section{Expression and Purification of $\mathrm{DnaE}_{\mathrm{C}}-\mathrm{GST}$}

E. coli BL21-Star (DE3) (Invitrogen) was transformed with vector DnaE ${ }_{C}-$ GST-pET29a. Medium 2xYT (Difco) containing $50 \mu \mathrm{g} / \mathrm{mL}$ kanamycin was inoculated with an overnight culture and grown at $37^{\circ} \mathrm{C}, 220 \mathrm{rpm}$. At an $\mathrm{OD}_{600}$ of 0.4 temperature was decreased to $16^{\circ} \mathrm{C}$. Protein expression was induced with $0.3 \mathrm{mM}$ IPTG at an $\mathrm{OD}_{600}$ of 0.8 . After 48 h cells were harvested $\left(3,000 \times \mathrm{g}, 20^{\prime}\right)$ and suspended in buffer $(500 \mathrm{mM} \mathrm{NaCl}, 50 \mathrm{mM}$ Tris- $\mathrm{HCl} \mathrm{pH}$ 7.5, $2 \mathrm{mM} \beta$-mercaptoethanol). Cell lysate was obtained by high pressure homogenization (Constant cell disruption systems, Constant Systems Ltd, UK) and subsequent centrifugation $\left(20,000 \times \mathrm{g}, 30\right.$ '). Purification of $\mathrm{DnaE}_{\mathrm{C}^{-}}$ GST protein was achieved by applying Glutathione Sepharose $(500 \mathrm{mM} \mathrm{NaCl}, 50 \mathrm{mM}$ Tris-HCl pH 7.5, $2 \mathrm{mM} \beta$-mercaptoethanol, elution with $10 \mathrm{mM}$ reduced glutathione), followed by gel filtration $(500 \mathrm{mM} \mathrm{NaCl}$, $50 \mathrm{mM}$ Hepes- $\mathrm{NaOH}$, pH 7.5).

\section{Intein catalyzed protein modification}

For GyrA mediated EPL, $5 \mu \mathrm{M}$ L19 IgG-GyrA was incubated in $500 \mathrm{mM} \mathrm{NaCl}, 50 \mathrm{mM}$ Hepes- $\mathrm{NaOH}, \mathrm{pH}$ 8.0, 50 $\mathrm{mM}$ Mesna (2-mercaptoethane sulfonate-sodium), $5 \mathrm{mM}$ cysteine or Bio-P1 Peptide (NEB). For DnaE mediated protein trans-splicing, $5 \mu \mathrm{M}$ L19 IgG-DnaE ${ }_{\mathrm{N}}$ was mixed with $50 \mu \mathrm{M}$ DnaE ${ }_{C}$ Peptide (MVKVIGRRSLGVQRIFDIGLPQDHNFLLANGAIAANCFN, kind gift from Prof. Christian Becker, TU Munich, Germany. The sequence corresponds to $\mathrm{DnaE}_{\mathrm{C}}$ from Synechocystis sp. strain 
PCC6803 (Ssp) which is interchangeable with $\mathrm{Npu} \mathrm{DnaE}_{\mathrm{C}}$ [32]), DnaE ${ }_{C}$-Biotin (MVKVIGRRSLGVQRIFDIGLPQDH NFLLANGAIAANCFNK-Biotin, synthesis by JPT Peptide Technologies $\mathrm{GmbH}$, Berlin, Germany) or $\mathrm{DnaE}_{\mathrm{C}^{-}}-\mathrm{GST}$ in $500 \mathrm{mM} \mathrm{NaCl}, 50 \mathrm{mM}$ Hepes- $\mathrm{NaOH}, \mathrm{pH} 7.5,5 \mathrm{mM}$ DTT. After incubation for 22-24h at room temperature (RT), intein activity was stopped by removing reducing agents (Mesna, DTT, cysteine) by dialysis over night at RT. For purification, the sample was loaded onto a Ni-IMAC column (500 mM NaCl, $50 \mathrm{mM}$ Hepes- $\mathrm{NaOH}, \mathrm{pH}$ 7.5). Completely processed L19 IgG was not in the flow through but was eluted at $30 \mathrm{mM}$ imidazole. Partly- or non-processed L19 IgG-GyrA or - DnaE $E_{N}$ was eluted with $300 \mathrm{mM}$ imidazole. For subsequent analysis, protein was dialyzed against $500 \mathrm{mM} \mathrm{NaCl}, 50 \mathrm{mM}$ Tris- $\mathrm{HCl}, \mathrm{pH} 7.5$ and concentrated to $1-2 \mathrm{mg} / \mathrm{mL}$.

\section{SEC}

A sample $(\sim 50 \mu \mathrm{g})$ was loaded onto a gel filtration column connected to an HPLC instrument (Agilent Technologies 1200 Series) equipped with detectors for refractive indices (RI) and multi angle light scattering (MALS) (Optilab rEX and miniDAWN TREOS, Wyatt Technology Corp., Santa Barbara, CA). To calculate the yield of intein processing, a sample after EPL/PTS but before purification was analyzed. The mass of the excised intein was determined by its RI signal and set into relation to the total loaded mass.

\section{Pepsin digestion and mass spectrometry}

L19 IgG was dialyzed against $20 \mathrm{mM} \mathrm{NaAcetat} \mathrm{pH} 4.5$. Pepsin (Protea Biosciences) was added to give an enzyme-to-antibody ratio of 1:25 (w:w) and incubated for $24 \mathrm{~h}$ at $37^{\circ} \mathrm{C}$. A sample was directly analyzed by ESITOF to assess masses of peptides derived by the Pepsin digest. Further samples were separated by reducing and non-reducing SDS-PAGE and gels were Coomassie stained. Protein bands were excised, de-stained, reduced with DTT, alkylated with iodacetamide and digested with trypsine. Protein sequences were determined by ESI-Q-TOF (MS and MS/MS) and search in Sequest (Finnigan) and Mascot Databases.

\section{Western blot}

After SDS-PAGE, gels were blotted onto a nitrocellulose membrane (iBlot, Invitrogen) and incubated for $1 \mathrm{~h}$ in blocking buffer (TBS with 0.05\% (v/v) Tween-20 and 5\% (w/v) milk powder). Biotin was detected by incubating with $1 \mu \mathrm{g} / \mathrm{ml}$ Streptavidin-Alkaline Phosphatase (Sigma) in blocking buffer over night. GST was detected by over night incubation with anti-GST antibody (GE Healthcare, 1:5000 in blocking buffer) followed by $1 \mathrm{~h}$ incubation with anti-Goat IgG-Alkaline Phosphatase (Sigma, 1:1000 in blocking buffer) as secondary antibody. BCIP/NBT (SigmaFAST tablet) was used for membrane staining.

\section{Surface Plasmon resonance (SPR)}

A Biacore T100 instrument (GE Healthcare, Munich) was employed to study the interaction between L19 IgG or modified versions thereof and ED-B, Fc $\gamma$ RI/CD64 and Fc $\gamma$ RIIIA/CD16a. L19 IgG or modified versions thereof were immobilized on a Biacore Sensor Chip (Series S Sensor Chip CM5 treated with antibody capture kit, GE Healthcare). Soluble ED-B domain in several concentrations $(1.56-200 \mathrm{nM})$ or soluble Fc receptor fragments Fc $\gamma$ RI/CD64 and Fc $\gamma$ RIIIA/CD16a (R\&D Systems) in several concentrations (1.56 - $50 \mathrm{nM}$ ) were used as analytes. Association and dissociation kinetics were detected to calculate the dissociation constant.

\section{Additional material}

Additional file 1: Table S1. Test of FC functionality after EPL and PTS.

The interaction with two Fcy receptors was analyzed by SPR.

\section{Acknowledgements}

The authors thank Prof. Dario Neri (Philogen) for providing the L19 single chain sequence and Prof. Christian Becker (TU Munich) for his initial support with the DnaE intein system and for synthesis of the DnaEc peptide. This study was funded by Bayer Healthcare, Berlin, Germany.

\section{Author details}

'Bayer Healthcare Research Center, Aprather Weg 18a, 42113 Wuppertal, Germany. ${ }^{2}$ Bayer HealthCare LLC, 455 Mission Bay Blvd South, San Francisco, CA 94158, USA. ${ }^{3}$ Bayer Healthcare, Nattermannallee 1, 50829 Cologne, Germany.

\section{Authors' contributions}

SM established the in vitro cleavage protocols, carried out protein expression, purification and analysis and wrote the manuscript. SG carried out the tryptic digest and the mass spectrometry. PB participated in the conceptional design of this project. AH supervised the research and contributed to the writing of the manuscript. All authors read and approved the final manuscript.

\section{Competing interests}

The authors are employees of Bayer Healthcare and declare that they have no competing interests.

Received: 27 October 2010 Accepted: 21 July 2011 Published: 21 July 2011

\section{References}

1. Chames P, Van Regenmortel M, Weiss E, Baty D: Therapeutic antibodies: successes, limitations and hopes for the future. Br J Pharmacol 2009, 157:220-233.

2. Johnson P, Glennie M: The mechanisms of action of rituximab in the elimination of tumor cells. Semin Oncol 2003, 30:3-8.

3. Sliwkowski MX, Lofgren JA, Lewis GD, Hotaling TE, Fendly BM, Fox JA: Nonclinical studies addressing the mechanism of action of trastuzumab (Herceptin). Semin Oncol 1999, 26:60-70.

4. Alinari L, Lapalombella R, Andritsos L, Baiocchi RA, Lin TS, Byrd JC: Alemtuzumab (Campath-1H) in the treatment of chronic lymphocytic leukemia. Oncogene 2007, 26:3644-3653.

5. Carter PJ, Senter PD: Antibody-drug conjugates for cancer therapy. Cancer J 2008, 14:154-169.

6. Menrad A, Menssen HD: ED-B fibronectin as a target for antibody-based cancer treatments. Expert Opin Ther Targets 2005, 9:491-500. 
7. Borsi L, Balza E, Bestagno M, Castellani P, Carnemolla B, Biro A, Leprini A Sepulveda J, Burrone O, Neri D, Zardi L: Selective targeting of tumoral vasculature: comparison of different formats of an antibody (L19) to the ED-B domain of fibronectin. Int J Cancer 2002, 102:75-85.

8. Santimaria M, Moscatelli G, Viale GL, Giovannoni L, Neri G, Viti F, Leprini A, Borsi L, Castellani P, Zardi L, Neri D, Riva P: Immunoscintigraphic detection of the ED-B domain of fibronectin, a marker of angiogenesis, in patients with cancer. Clin Cancer Res 2003, 9:571-579.

9. Sauer S, Erba PA, Petrini M, Menrad A, Giovannoni L, Grana C, Hirsch B, Zardi L, Paganelli G, Mariani G, Neri D, Dürkop H, Menssen HD: Expression of the oncofetal ED-B-containing fibronectin isoform in hematologic tumors enables ED-B-targeted 131I-L19SIP radioimmunotherapy in Hodgkin lymphoma patients. Blood 2009, 113:2265-2274

10. Johannsen M, Spitaleri G, Curigliano G, Roigas J, Weikert S, Kempkensteffen C, Roemer A, Kloeters C, Rogalla P, Pecher G, Miller K, Berndt A, Kosmehl H, Trachsel E, Kaspar M, Lovato V, González-Iglesias R, Giovannoni L, Menssen HD, Neri D, de Braud F: The tumour-targeting human L19-IL2 immunocytokine: Preclinical safety studies, phase I clinical trial in patients with solid tumours and expansion into patients with advanced renal cell carcinoma. Eur J Cancer 2010.

11. Wu AM, Senter PD: Arming antibodies: prospects and challenges for immunoconjugates. Nat Biotechnol 2005, 23:1137-1146.

12. Hamblett KJ, Senter PD, Chace DF, Sun MMC, Lenox J, Cerveny CG, Kissler KM, Bernhardt SX, Kopcha AK, Zabinski RF, Meyer DL, Francisco JA: Effects of drug loading on the antitumor activity of a monoclonal antibody drug conjugate. Clin Cancer Res 2004, 10:7063-7070.

13. McDonagh CF, Turcott E, Westendorf L, Webster JB, Alley SC, Kim K, Andreyka J, Stone I, Hamblett K, Francisco JA, Carter P: Engineered antibody-drug conjugates with defined sites and stoichiometries of drug attachment. Protein Eng Des Sel 2006, 19:299-307.

14. Junutula JR, Raab H, Clark S, Bhakta S, Leipold DD, Weir S, Chen Y, Simpson M, Tsai SP, Dennis MS, Lu Y, Meng YG, Ng C, Yang J, Lee CC, Duenas E, Gorrell J, Katta V, Kim A, McDorman K, Flagella K, Venook R, Ross S, Spencer SD, Lee Wong W, Lowman HB, Vandlen R, Sliwkowski MX, Scheller RH, Polakis P, Mallet W: Site-specific conjugation of a cytotoxic drug to an antibody improves the therapeutic index. Nat Biotechnol 2008, 26:925-932.

15. O'Shannessy DJ, Quarles RH: Labeling of the oligosaccharide moieties of immunoglobulins. J Immunol Methods 1987, 99:153-161.

16. Stan AC, Radu DL, Casares S, Bona CA, Brumeanu TD: Antineoplastic efficacy of doxorubicin enzymatically assembled on galactose residues of a monoclonal antibody specific for the carcinoembryonic antigen. Cancer Res 1999, 59:115-121.

17. Scheck RA, Francis MB: Regioselective labeling of antibodies through $\mathrm{N}$ terminal transamination. ACS Chem Biol 2007, 2:247-251

18. Wu P, Shui W, Carlson BL, Hu N, Rabuka D, Lee J, Bertozzi CR: Site-specific chemical modification of recombinant proteins produced in mammalian cells by using the genetically encoded aldehyde tag. Proc Natl Acad Sci USA 2009, 106:3000-3005.

19. Kaneko Y, Nimmerjahn F, Ravetch JV: Anti-inflammatory activity of immunoglobulin G resulting from Fc sialylation. Science 2006, 313:670-673

20. Sunbul $M$, Yin J: Site specific protein labeling by enzymatic posttranslational modification. Org Biomol Chem 2009, 7:3361-3371.

21. Saleh L, Perler FB: Protein splicing in cis and in trans. Chem Rec 2006, 6:183-193.

22. Muir TW, Sondhi D, Cole PA: Expressed protein ligation: A general method for protein engineering. Proc Natl Acad Sci USA 1998, 95:6705-6710

23. Muir TW: Semisynthesis of proteins by expressed protein ligation. Annu Rev Biochem 2003, 72:249-289.

24. $\mathrm{Wu} \mathrm{H}, \mathrm{Hu}$ Z, Liu XQ: Protein trans-splicing by a split intein encoded in a split DnaE gene of Synechocystis sp. PCC6803. Proc Natl Acad Sci USA 1998, 95:9226-9231.

25. Evans TC Jr, Martin D, Kolly R, Panne D, Sun L, Ghosh I, Chen L, Benner J, Liu XQ, Xu MQ: Protein trans-splicing and cyclization by a naturally split intein from the dnaE gene of Synechocystis species PCC6803. J Biol Chem 2000, 275.9091-9094

26. Mootz HD: Split inteins as versatile tools for protein semisynthesis. Chembiochem 2009, 10:2579-2589.
27. Sydor $J R$, Mariano M, Sideris S, Nock S: Establishment of intein-mediated protein ligation under denaturing conditions: C-terminal labeling of a single-chain antibody for biochip screening. Bioconjug Chem 2002, 13:707-712.

28. Reulen SWA, van Baal I, Raats JMH, Merkx M: Efficient, chemoselective synthesis of immunomicelles using single-domain antibodies with a Cterminal thioester. BMC Biotechnol 2009, 9:66.

29. Chaisemartin L, Chinestra P, Favre G, Blonski C, Faye JC: Synthesis and application of a $\mathrm{N}-1$ ' fluorescent biotinyl derivative inducing the specific carboxy-terminal dual labeling of a novel RhoB-selective scFv. Bioconjug Chem 2009, 20:847-855.

30. Telenti A, Southworth M, Alcaide F, Daugelat S, Jacobs WR, Perler FB: The Mycobacterium xenopi GyrA protein splicing element: characterization of a minimal intein. J Bacteriol 1997, 179:6378-6382.

31. Evans TC, Benner J, Xu MQ: Semisynthesis of cytotoxic proteins using a modified protein splicing element. Protein Sci 1998, 7:2256-2264.

32. Iwai H, Züger S, Jin J, Tam PH: Highly efficient protein trans-splicing by a naturally split DnaE intein from Nostoc punctiforme. FEBS Lett 2006, 580:1853-1858

33. Aranko AS, Züger $S$, Buchinger $E$, Iwaï $H$ : In vivo and in vitro protein ligation by naturally occurring and engineered split DnaE inteins. PLOS ONE 2009, 4:e5185.

34. Andrew SM, Titus JA: Fragmentation of immunoglobulin G. Curr Protoc Cell Biol 2003, Chapter 16:Unit 16.4

35. Pastan I, Hassan R, FitzGerald DJ, Kreitman RJ: Immunotoxin treatment of cancer. Annu Rev Med 2007, 58:221-237.

36. Pini A, Viti F, Santucci A, Carnemolla B, Zardi L, Neri P, Neri D: Design and use of a phage display library. Human antibodies with subnanomolar affinity against a marker of angiogenesis eluted from a two-dimensional gel. J Biol Chem 1998, 273:21769-21776.

37. Durocher $Y$, Perret S, Kamen A: High-level and high-throughput recombinant protein production by transient transfection of suspensiongrowing human 293-EBNA1 cells. Nucleic Acids Res 2002, 30:E9.

doi:10.1186/1472-6750-11-76

Cite this article as: Möhlmann et al: Site-specific modification of ED-Btargeting antibody using intein-fusion technology. BMC Biotechnology $201111: 76$

\section{Submit your next manuscript to BioMed Central and take full advantage of:}

- Convenient online submission

- Thorough peer review

- No space constraints or color figure charges

- Immediate publication on acceptance

- Inclusion in PubMed, CAS, Scopus and Google Scholar

- Research which is freely available for redistribution

Submit your manuscript at www.biomedcentral.com/submit
C Biomed Central 